\title{
Research on Management Service Platform for Micro-application
}

\author{
Zhang $X u^{1}$, Wang Jianfeng ${ }^{2}$, Wang Qiang ${ }^{1}$, Tian Tian ${ }^{1}$ \\ ${ }^{1}$ State Grid Tianjin Electric Power Company, Tianjin City, Tianjin City 300200 \\ ${ }^{2}$ State Grid Tianjin Electric Power Company
}

Keywords: micro-application; flew object; template customization; master data.

\begin{abstract}
The research of the platform breaks the existing project development model. Through the establishment of a unified platform, the unified management mode of job-oriented work standards and work processes is realized. Various professional departments use the platform to build "micro-applications" that meet their own needs. This paper in detail planned the flow processing object as the core processing unit, took business management flow as a breakthrough, standardized process management as the main body, and formed a micro-application management model to achieve various "micro-application" examples to build and put into operation and maintenance of the entire process of standardized operation management.
\end{abstract}

\section{Introduction}

With the advancement of informatization technology, the informatization construction of each group company is evolving in the direction of integration and integration. This brings with it the following problems: 1 Due to the problems of management boundaries, repeated investment is caused, and the amount of each investment is huge; 2 Business changes lead to process adjustments, which require partial adjustment of the system, which may affect the stability, reliability, and significant increase in adjustment costs of the entire system. 3 Due to the needs of specific business, all subsidiaries need to build systems to varying degrees. The self-built system faces challenges in effective management of hardware input costs, software deployment costs, development costs, and operation and maintenance costs.

In order to solve the above problems, we put forward the idea of "Micro-application", which includes the self-built system into the scope of unified configuration management. The development is centered on micro-application, and it has "computerized" rapid development technology, flexible business process management technology and complex history. The application platform for data reuse technology.

(1) Through the research of the platform, the original project development model was broken, and the informatization development ideas of "big platform, micro-application, and componentization" formulated by the State Grid Corporation were fully reflected, and an inter-departmental and multi-professional business process management platform was established. Realize the close integration of business data and business processes, and achieve the sharing and integration of business applications and business data across professions.

(2) Through the establishment of a unified platform, a unified management model for job-oriented work standards and work processes is implemented [1]. Each professional department uses the platform and builds a "micro-application" that meets its own needs according to the constraints of the platform and realizes the simplified, distributed, and unified management ofbusiness processes, business data.

(3) Through the research of this platform, it simplifies the management mode, clears the way of collection of multiple data, realizes convenience, flexible collection, diagnosis, processing, and improvement of various types of data, and achieves horizontal and vertical full penetration of business data, combined with big data processing technology [2], fully serves the requirements of business management. 


\section{System Structure}

Take business process management as a breakthrough, study the characteristics of each business process, extract core points from it, and analyze it from the perspective of information implementation to form a model framework for business process management [3-5].

Taking the circulation object as the core processing unit, taking the flow of business management as a breakthrough point, and adopting standardized process management as the main body, it forms the management model of the micro-application platform and realizes the standard operation management of the whole process of setting up, putting into operation, and operation and maintenance of application instances.

Taking the informatization management of business processes as a breakthrough, with the aim of standardization and flexibility of process realization, the unified management mechanism of business processes is studied to ensure the smooth flow of data collection, verification, analysis, and aggregation channels.

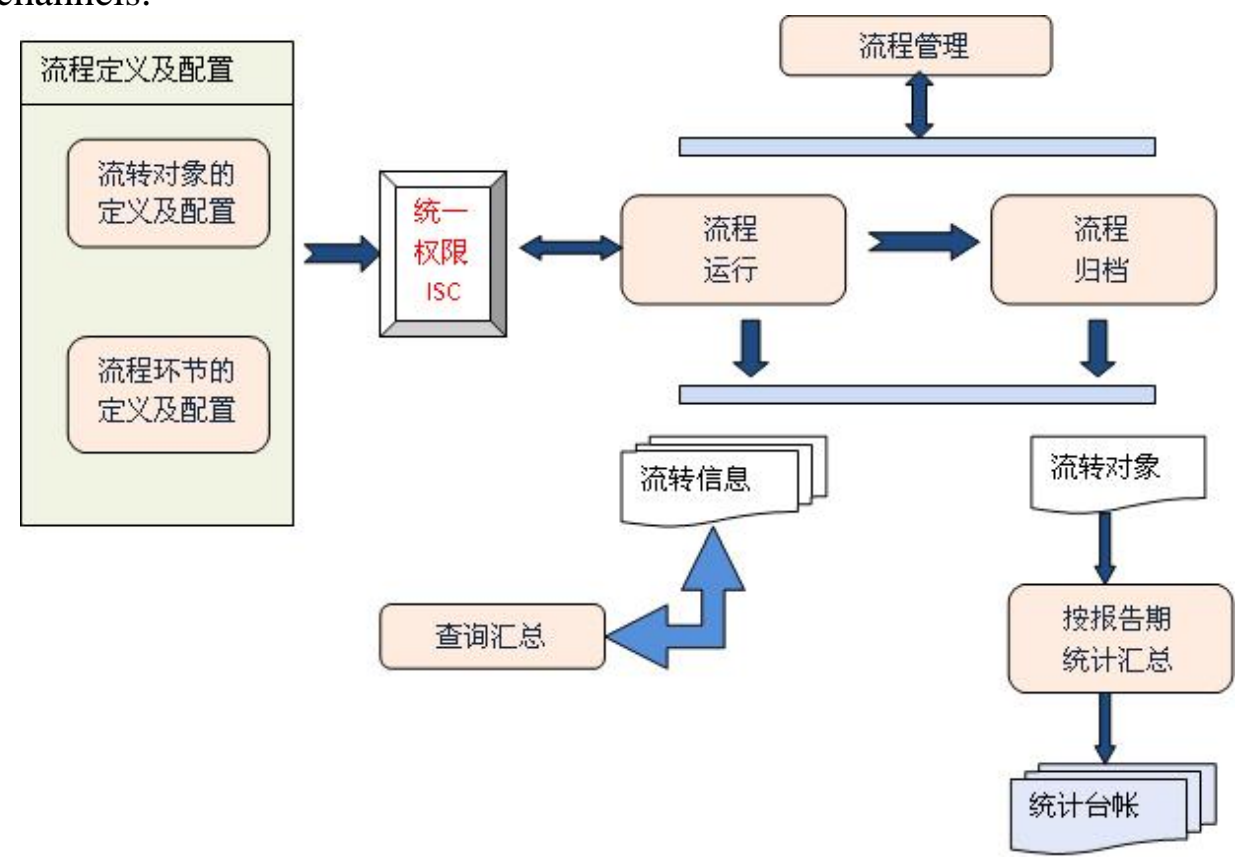

Fig.1 System architecture

\section{Core Technology Research}

\subsection{Process Definition and Configuration}

On the basis of satisfying the normal operation of the business process, the process definition and configuration process fully reflect the characteristics of rapidness, flexibility and completeness. The entire process definition and configuration is divided into two parts: the definition and configuration of the transfer object, and the definition and configuration of the check procedure.

(1) Definition and configuration of transfer objects

The purpose of the process operation is to make sure the integrity of various types of data collection. Therefore, in the entire process definition and configuration process, the planning of the transfer object $^{[6]}$ directly affects the relationship between the enterprise's organizational structure, information system, and production practices.

Research on the transfer of objects focuses on the following aspects:

1) Due to the need for business development and the requirements of process diversity, we have proposed a template-based approach to achieve flexible customization of transfer objects.

2) Research on the diversity of transfer objects: data types are subdivided into structured data and non-structural data. Data collection is completed through the routine introduction of structural data. For non-structural data, a structured index is used to establish associations with structured data. The rational classification and storage of unstructured data is realized by the mechanism of 
structured data management.

3) Version management of transfer objects: During the process of running the transfer objects, local adjustment may occur. After the adjustment, management is performed according to separate version, and data storage and update management mechanism is established through version control.

(2) Definition of process links

In order to ensure the concise and intuitive definition of the process links, the visual process customization tools are used to realize the WYSIWYGmanagement effect.

Through the graphical process customization tool, the customization of the entire process node has been completed. The definition of process node and configuration process embodies the following features:

Table 1 The process node configuration table

\begin{tabular}{|c|c|c|}
\hline \multicolumn{3}{|c|}{ Definition of Process node and configuration characteristics } \\
\hline The node type & Start/end nodes & \\
\hline & $\begin{array}{l}\text { Department node } \\
\text { Global node } \\
\text { branch node } \\
\text { sub-process }\end{array}$ & $\begin{array}{l}\text { Establish close cooperation with } \\
\text { the main process }\end{array}$ \\
\hline \multirow{4}{*}{ technical index } & Merge Node & \\
\hline & $\begin{array}{l}\text { Define the WYSIWYG } \\
\text { process }\end{array}$ & \\
\hline & $\begin{array}{l}\text { Automatic binding with } \\
\text { the unified permission } \\
\text { management platform }\end{array}$ & \\
\hline & $\begin{array}{l}\text { Version control of the } \\
\text { flow object }\end{array}$ & \\
\hline
\end{tabular}

\subsection{Process Operation}

Through the establishment of a unified business center, the operational status of each process is intuitively and comprehensively reflected, and the business personnel are guided to complete relevant work tasks within the time limit required by the periodicity.

Under the control of the unified rights management, the relevant processes that need to be handled are automatically sifted and notified via the "remind" method [7]. And the implementation of the process is accomplished under strict authority control.

Throughout the whole process, we have focused on solving the following issues:

(1) Support diversity of data collection methods: In order to facilitate the collection of data and reduce the workload of business personnel, it is necessary to study the access methods of various electronic files, such as TXT files, WPS files, Excel files, XML files, etc. The possibility of structural transformation can be achieved by analyzing different data interfaces.

(2) The establishment of the effectiveness detection mechanismof the collected data: The effectiveness of data collection is built on a specified range of rules to avoid unnecessary errors in the data

(3) Assessment of process execution: Through the control of processing node's execution time limit, a judicious evaluation of process execution efficiency, and assessment of the enthusiasm of the business staff.

\subsection{Process Archive}

After the process is expected to be completed, the process is archived to accomplish the data update. In the process of archiving the entire process, the following issues were focused on:

(1) Data update mechanism ${ }^{[8]}$ : Analyze the data of the transfer object according to the template, perform the necessary format verification, and complete the update of the data.

(2) The conversion of structured data and unstructured data: For the archived data, in order to 
save the convenience and its unchangeable characteristic requirements, it is necessary to study the characteristics of related unstructured data, and to make its structured data unstructured. Conversion.

\subsection{Process Management}

Process management includes: management of operational processes and management of archiving processes. Owing to their different characteristics and diverse business requirements, it is necessary to explore different management models ${ }^{[8-10]}$.

(1) Operational process management: Through the real-time monitoring of the implementation of each process to understand its status, according to the requirements can force change the status of the relevant processes, including: forcibly return the process to the relevant node, the node to supplement processing; can be forced Insert a node, which is supplemented by related personnel; it can also force the process to terminate.

(2) Archiving process management: archiving process according to the degree of importance, according to the time limit to retain a period of time, when the time limit reaches a certain condition, to remind the completion of the archiving process to clean up, in order to redistribute software and hardware resources, improve its application efficiency.

\subsection{Data Summary Unit}

The data summary unit considers feasible and comprehensiveness, and R\&D focuses on the stati stical summary of the circulation objects. The statistic of circulation objects focuses on the statistica $\mathrm{l}$ analysis of structured data. Built on the data of the reporting period, it automatically creates electro nic statistical ledgers and completes the data collection of different calibers in different reporting pe riods. The reporting period can be divided into: day, month, season, year, and so on.

\section{Conclusion}

To instantiate this achievement and establish a complete set of platforms for collecting, sorting, sharing, summarizing, statistics, and output presentation of data from horizontal and vertical aspects. Automated management of business processes will surely bring considerable social and economic benefits to the enterprise in terms of information management.

(1) Through the research of the project, and through the unified business process management platform, business applications and business data are shared and integrated across disciplines, and the scope of building a self-built system is reduced to a large extent, thereby saving hardware input costs, software deployment costs, development costs, operation and maintenance costs, etc.

(2) Standardized management of business processes became possible, avoiding the phenomenon of non-conformity between the current regulations and actual management in the current management system.

(3) Controllability of data security: Since data management fully complies with the standardization requirements. Data access is under strict and uniform platform access control, and therefore security is effectively guaranteed ${ }^{[11]}$.

(4) Effective management of main data, rapid definition of business processes, efficient configuration, flexible setting of business data, and seamless connection with other technology platforms, which ensure that the data acquisition is reliable, fast and accurate, and on the other hand, which reduce the difficulty of operation and maintenance personnel and improves the efficiency of operation and maintenance.

\section{References}

[1] JIA Nan, LI Dong, ZHOU Qiang. Construction of an information operation and maintenance standardized workflow with the "five-in-one" mode [J].Electric Power Information and Communication Technology. 2015, 13(10):104- 107.

[2] WANG De-wen, Basic framework and key technology for a new generation of data center in 
electric power corporation based on cloud computation [J]. Automation of Electric Power System. 2012.36(11):67-71.

[3] Zhang Bing. Service-oriented e-commerce platform centralized operation and maintenance management practices [J]. Electric Power Information and Communication Technology, 2015, 13 (9): 116-119.

[4] Zhang Bing.Practice op centralized operation and maintenance management of service-oriented electronic commerce platform [J].Electric Power Information and Communication Technology, 2015, 13(9):116-119.

[5] Wang Lei. Architecture and Practice of Microservices [M]. Beijing: Publishing House of Electronics Industry, 2015:13-38

[6] Zhang Bing. Service-oriented e-commerce platform centralized operation and maintenance management practices [J]. Power Information and Communication Technology, 2015, 13 (9):116-119.

[7] Zhang Bing.Practice op centralized operation and maintenance management of service-oriented electronic commerce platform [J].Electric Power Information and Communication Technology, 2015, 13(9) :116-119.

[8] REEVE A. Data Integration Technologies, Methods, and Best Practices [M]. Beijing: Machinery Industry Press, 2014.

[9] Xu Shangying. Research on intelligent discovery method of network services for user's personalized demand [D]. Wuhan University of Technology, 2014.

[10] Luo Junzhou, Jin Jiahui, Song Aibo, et al. Cloud computing: architecture and key technologies [J]. Journal of Communications, 2011, 32(7) :18-21.

[11] LUO Jun-zhou, JIN Jia-hui, SONG Ai-bo, et al.Cloud computing: architecture and key technologies [J].Journal on Communications, 2011,32(7) :18-21.

[12] DU Baoli. Design and Implementation of Cloud Database Service System Based on Docker [D]. Xidian University, 2015.

[13] He Songlin. Research on the construction of elastic cluster built on Docker resource pre-scheduling strategy [D]. Zhejiang Sci-Tech University, 2017.

[14] Li Ruifeng. Research on Mobile Analysis Application and Security Mechanism in Electric Power Enterprises [J]. Information and Communications, 2014(1): 200-201

[15] LI Rui-feng. Research on mobile analysis applications and security mechanisms in electric power enterprises [J].Information \& Communications. 2014(1):200-201 